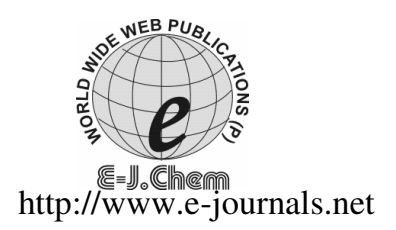

ISSN: 0973-4945; CODEN ECJHAO

E-Journal of Chemistry

2011, 8(3), 1000-1005

\title{
Molecular Iodine: A Versatile Catalyst for the Synthesis of $2 H$-indazolo[2,1-b]phthalazine- 1,6,11(13H)-trione Derivatives in Ethanol
}

\author{
XIAO WANG, GUANGZHOU LU, WEIWEI MA* and LIQZANG WU \\ School of Pharmacy, Xinxiang Medical University \\ Xinxiang, Henan - 453003, P. R. China \\ weiwei525626@163.com
}

Received 22 July 2010; Accepted 15 October 2010

\begin{abstract}
An efficient method for the synthesis of $2 H$-indazolo[2,1- $b]$ phthalazine-1,6,11(13H)-trione derivatives by a three-component condensation reaction of dimedone, aromatic aldehydes and phthalhydrazide in the presence of a catalytic amount of molecular iodine in ethanol is described.
\end{abstract}

Keywords: $2 H$-indazolo[2,1- $b]$ phthalazine, Dimedone, Phthalhydrazide, Molecular iodine

\section{Introduction}

Phthalazine derivatives have attracted considerable attention in recent years because of their wide range of pharmaceutical activities such as antimicrobial ${ }^{1}$, anticonvulsant ${ }^{2}$, antifungal ${ }^{3}$, anticancer ${ }^{4}$, and anti-inflammatory ${ }^{5}$ activities. Therefore, a number of methods have been reported for the synthesis of phthalazine derivatives ${ }^{6}$. Nevertheless the development of new synthetic methods for the efficient preparation of heterocycles containing phthalazine ring fragment is therefore an interesting challenge. Recently, synthesis of $2 \mathrm{H}$-indazolo[2,1-b]phthalazine- 1,6,11(13H)-trione derivatives have been reported ${ }^{7-11}$ using $p$-TSA, $\mathrm{Me}_{3} \mathrm{SiCl}$, silica sulfuric acid, $\mathrm{H}_{2} \mathrm{SO}_{4}, \mathrm{Mg}\left(\mathrm{HSO}_{4}\right)_{2}$ and silica supported poly phosphoric acid ${ }^{12}$ as catalysts. However, many of these methodologies are associated with one or more disadvantages such as use of expensive catalyst or toxic organic solvents, strong acidic conditions and harsh reaction conditions.

In recent years, the usage of molecular iodine has drawn considerable attention as an inexpensive, nontoxic, readily available catalyst for various organic transformations to afford the corresponding products in excellent yields with high selectivity. The mild Lewis acidity associated with iodine enhances its usage in organic synthesis to realize several organic transformations using stoichiometric levels or even catalytic amounts ${ }^{13}$. As a part of our studies to explore the utility of iodine-catalyzed reactions ${ }^{14}$, we proceeded to examine the synthesis of $2 H$-indazolo[2,1-b]phthalazine-1,6,11(13H)-trione derivatives in the presence of molecular iodine in ethanol (Scheme 1). 
<smiles>CC1(C)[CH-]C(=O)CC(=O)C1</smiles>

1<smiles>O=c1[nH][nH]c(=O)c2ccccc12</smiles><smiles>[R]C1C2=C(CC(C)(C)CC2=O)n2c(=O)c3ccccc3c(=O)n21</smiles>

4

\section{Experimental}

Scheme 1

NMR spectra were determined on Bruker AV-400 spectrometer at room temperature using TMS as internal standard, coupling constants $(J)$ were measured in $\mathrm{Hz}$; Elemental analysis were performed by a Vario-III elemental analyzer; Melting points were determined on a XT-4 binocular microscope and were uncorrected; commercially available reagents were used throughout without further purification unless otherwise stated.

To a mixture of dimeone $(1 \mathrm{mmol})$, aldehyde $(1.2 \mathrm{mmol})$, phthalhydrazide $(1 \mathrm{mmol})$, and ethanol $(10 \mathrm{~mL}), \mathrm{I}_{2}(0.1 \mathrm{mmol})$ was added. The mixture was stirred at reflux for the appropriate time ( $c f$.Table 3). After completion of the reaction (TLC), the mixture was treated with aqueous $\mathrm{Na}_{2} \mathrm{~S}_{2} \mathrm{O}_{3}$ solution, extracted with $\mathrm{CH}_{2} \mathrm{Cl}_{2}(2 \times 10 \mathrm{~mL})$, filtered and the solvent evaporated in vacuo. Products 4 were purified by recrystallizing from aqueous ethanol $(25 \%)$.

\section{3,4-Dihydro-3,3-dimethyl-13-phenyl-2H-indazolo[2,1-b]phthalazine-1,6,11(13H)-} trione (4a)

${ }^{1} \mathrm{H}$ NMR $\left(\mathrm{CDCl}_{3}, 400 \mathrm{MHz}\right) \delta: 8.37-8.27(\mathrm{~m}, 2 \mathrm{H}), 7.86(\mathrm{dd}, 2 \mathrm{H}, J=3.2,7.6 \mathrm{~Hz}), 7.42$ $(\mathrm{d}, 2 \mathrm{H}, J=7.2 \mathrm{~Hz}), 7.37-7.29(\mathrm{~m}, 3 \mathrm{H}), 6.46(\mathrm{~s}, 1 \mathrm{H}), 3.43(\mathrm{~d}, 1 \mathrm{H}, J=18.8 \mathrm{~Hz}), 3.25(\mathrm{dd}, 1 \mathrm{H}$, $J=2.4,18.8 \mathrm{~Hz}), 2.35(\mathrm{~s}, 2 \mathrm{H}), 1.22(\mathrm{~s}, 6 \mathrm{H}) ;{ }^{13} \mathrm{C} \mathrm{NMR}\left(\mathrm{CDCl}_{3}, 100 \mathrm{MHz}\right) \delta: 192.1,156.0$, $154.3,150.8,136.4,134.5,133.5,129.1,129.0,128.7,128.0,127.7,127.1,118.6,65.0$, 50.9, 38.0, 34.6. 28.7, 28.5; MS (ESI) $\mathrm{m} / \mathrm{z} 373(\mathrm{M}+1)$; Anal. calcd for $\mathrm{C}_{23} \mathrm{H}_{20} \mathrm{~N}_{2} \mathrm{O}_{3}$ : C 74.18, H 5.41, N 7.52; found: C 74.25, H 5.36, N 7.48.

\section{3,4-Dihydro-3,3-dimethyl-13-(4-chlorophenyl)-2H-indazolo[2,1-b]phthalazine-1,6, $11(13 H)$-trione $(\mathbf{4 b})$}

${ }^{1} \mathrm{H}$ NMR $\left(\mathrm{CDCl}_{3}, 400 \mathrm{MHz}\right) \delta: 8.38-8.26(\mathrm{~m}, 2 \mathrm{H}), 7.88-7.85(\mathrm{~m}, 2 \mathrm{H}), 7.37(\mathrm{~d}, 2 \mathrm{H}, J=8.4 \mathrm{~Hz})$, $7.31(\mathrm{~d}, 2 \mathrm{H}, J=8.4 \mathrm{~Hz}), 6.42(\mathrm{~s}, 1 \mathrm{H}), 3.41(\mathrm{~d}, 1 \mathrm{H}, J=18.8 \mathrm{~Hz}), 3.25(\mathrm{dd}, 1 \mathrm{H}, J=2.0$, $18.8 \mathrm{~Hz}), 2.35(\mathrm{~s}, 2 \mathrm{H}), 1.27-1.21(\mathrm{~m}, 6 \mathrm{H}) ;{ }^{13} \mathrm{C} \mathrm{NMR}\left(\mathrm{CDCl}_{3}, 100 \mathrm{MHz}\right) \delta: 192.1,156.0$, $154.4,151.1,134.9,134.6,134.5,133.7,129.0,128.9,128.5,128.0,127.7,118.0,64.3$, 50.9, 38.0, 34.7, 28.7, 28.4; MS (ESI) $\mathrm{m} / \mathrm{z} 407(\mathrm{M}+1)$; Anal. calcd for $\mathrm{C}_{23} \mathrm{H}_{19} \mathrm{ClN}_{2} \mathrm{O}_{3}$ : C 67.90, H 4.71, N 6.89; found: C 67.95, H 4.82, N 6.79.

\section{3,4-Dihydro-3,3-dimethyl-13-(4-methoxylphenyl)-2H-indazolo[2,1-b]phthalazine-1,6,} 11(13H)-trione $(\mathbf{4 c})$

${ }^{1} \mathrm{H} \mathrm{NMR}\left(\mathrm{CDCl}_{3}, 400 \mathrm{MHz}\right) \delta: 8.38-8.26(\mathrm{~m}, 2 \mathrm{H}), 7.86-7.83(\mathrm{~m}, 2 \mathrm{H}), 7.35(\mathrm{~d}, 2 \mathrm{H}, J=8.8 \mathrm{~Hz})$, $6.86(\mathrm{~d}, 2 \mathrm{H}, J=8.4 \mathrm{~Hz}), 6.43(\mathrm{~s}, 1 \mathrm{H}), 3.77(\mathrm{~s}, 3 \mathrm{H}), 3.43(\mathrm{~d}, 1 \mathrm{H}, J=18.8 \mathrm{~Hz}), 3.24$ $(\mathrm{dd}, 1 \mathrm{H}, J=2.0,18.8 \mathrm{~Hz}), 2.35(\mathrm{~s}, 2 \mathrm{H}), 1.29-1.22(\mathrm{~m}, 6 \mathrm{H}) ;{ }^{13} \mathrm{C} \mathrm{NMR}\left(\mathrm{CDCl}_{3}, 100\right.$ MHz) $\delta: 192.2,159.7,156.0,154.2,150.7,134.4,133.4,129.1,128.9,128.5,128.3$, $127.9,127.7,118.5,114.1,64.6,55.2,51.0,38.0,34.6,28.7,28.5$; MS (ESI) $\mathrm{m} / \mathrm{z} 403$ $(\mathrm{M}+1)$; Anal. calcd for $\mathrm{C}_{24} \mathrm{H}_{22} \mathrm{~N}_{2} \mathrm{O}_{4}$ : C 71.63, H 5.51, N 6.96; found: C 71.59, H 5.62, N 7.02 
3,4-Dihydro-3,3-dimethyl-13-(4-methylphenyl)-2H-indazolo[2,1-b]phthalazine-1,6, 11(13H)-trione $(\mathbf{4 d})$

${ }^{1} \mathrm{H} \mathrm{NMR}\left(\mathrm{CDCl}_{3}, 400 \mathrm{MHz}\right) \delta: 8.37-8.27(\mathrm{~m}, 2 \mathrm{H}), 7.87-7.83(\mathrm{~m}, 2 \mathrm{H}), 7.31(\mathrm{~d}, 2 \mathrm{H}, J=8.0 \mathrm{~Hz}), 7.15$ $(\mathrm{d}, 2 \mathrm{H}, J=7.6 \mathrm{~Hz}), 6.43(\mathrm{~s}, 1 \mathrm{H}), 3.43(\mathrm{~d}, 1 \mathrm{H}, J=18.8 \mathrm{~Hz}), 3.25(\mathrm{dd}, 1 \mathrm{H}, J=2.0,18.8 \mathrm{~Hz}), 2.34$ (s, 2H), 1.22 (s, 6H); ${ }^{13} \mathrm{C} \mathrm{NMR}\left(\mathrm{CDCl}_{3}, 100 \mathrm{MHz}\right) \delta: 192.2,156.0,154.2,150.7,138.5,134.4,133.4$, 133.3, 129.4, 129.1, 127.9, 127.7, 118.7, 64.8, 51.0, 38.0, 34.6, 28.7, 28.4, 21.2; MS (ESI) $\mathrm{m} / z .387$ (M+1); Anal. calcd for $\mathrm{C}_{24} \mathrm{H}_{22} \mathrm{~N}_{2} \mathrm{O}_{3}$ C 74.59, H 5.74, N 7.25; found: C 74.62, H 5.69, N 7.37.

3,4-Dihydro-3,3-dimethyl-13--(4-nitrophenyl)-2H-indazolo[2,1-b]phthalazine-1,6, 11(13H)-trione (4e)

${ }^{1} \mathrm{H}$ NMR $\left(\mathrm{CDCl}_{3}, 400 \mathrm{MHz}\right) \delta: 8.40-8.24(\mathrm{~m}, 2 \mathrm{H}), 8.21(\mathrm{~d}, 2 \mathrm{H}, J=8.8 \mathrm{~Hz}), 7.90(\mathrm{dd}, 2 \mathrm{H}$, $J=1.6,5.6 \mathrm{~Hz}), 7.62(\mathrm{~d}, 2 \mathrm{H}, J=8.8 \mathrm{~Hz}), 6.52(\mathrm{~s}, 1 \mathrm{H}), 3.42(\mathrm{~d}, 1 \mathrm{H}, J=19.2 \mathrm{~Hz}), 3.27$ $(\mathrm{dd}, 1 \mathrm{H}, J=2.0,19.2 \mathrm{~Hz}), 2.34(\mathrm{~s}, 2 \mathrm{H}), 2.31(\mathrm{~s}, 3 \mathrm{H}), 1.29-1.20(\mathrm{~s}, 6 \mathrm{H}) ;{ }^{13} \mathrm{C} \mathrm{NMR}\left(\mathrm{CDCl}_{3}\right.$, $100 \mathrm{MHz}) \delta$ : 192.0, 155.9, 154.5, 151.6, 147.9, 143.4, 134.8, 133.9, 128.9, 128.6, 128.2, 128.0, 127.8, 124.0, 117.3, 64.1, 50.8, 38.0, 34.7, 28.7, 28.4; MS (ESI) $\mathrm{m} / \mathrm{z} 418(\mathrm{M}+1)$; Anal. calcd for $\mathrm{C}_{23} \mathrm{H}_{19} \mathrm{~N}_{3} \mathrm{O}_{5}$ : C 66.18, H 4.59, N 10.07; found: C 66.21, H 4.50, N 10.01.

3,4-Dihydro-3,3-dimethyl-13--(3-nitrophenyl)-2H-indazolo[2,1-b]phthalazine-1,6, $11(13 H)$-trione $(\mathbf{4 f})$

${ }^{1} \mathrm{H}$ NMR $\left(\mathrm{CDCl}_{3}, 400 \mathrm{MHz}\right) \delta: 8.41-8.25(\mathrm{~m}, 2 \mathrm{H}), 8.18(\mathrm{~d}, 2 \mathrm{H}, J=7.2 \mathrm{~Hz}), 7.92-7.89$ $(\mathrm{m}, 3 \mathrm{H}), 7.57(\mathrm{t}, 1 \mathrm{H}, J=7.2 \mathrm{~Hz}), 6.54(\mathrm{~s}, 1 \mathrm{H}), 3.45(\mathrm{~d}, 1 \mathrm{H}, J=19.6 \mathrm{~Hz}), 3.29(\mathrm{dd}, 1 \mathrm{H}$, $J=2.0,19.6 \mathrm{~Hz}), 2.36(\mathrm{~s}, 2 \mathrm{H}), 1.27-1.19(\mathrm{~s}, 6 \mathrm{H}) ;{ }^{13} \mathrm{C} \mathrm{NMR}\left(\mathrm{CDCl}_{3}, 100 \mathrm{MHz}\right) \delta: 192.1$, 156.0, 154.6, 151.8, 148.5, 138.6, 134.8, 134.2, 133.9, 129.7, 129.0, 128.6, 128.2, 127.7, 123.7, 121.5, 117.1, 64.1, 50.8, 38.0, 34.7, 28.7, 28.4; MS (ESI) $\mathrm{m} / z .18(\mathrm{M}+1)$; Anal. calcd for $\mathrm{C}_{23} \mathrm{H}_{19} \mathrm{~N}_{3} \mathrm{O}_{5}$ : C 66.18, H 4.59, N 10.07; found: C 66.18, H 4.65, N 10.05.

3,4-Dihydro-3,3-dimethyl-13--(4-fluorophenyl)-2H-indazolo[2,1-b]phthalazine-1,6, 11(13H)-trione $(\mathbf{4 g})$

${ }^{1} \mathrm{H}$ NMR $\left(\mathrm{CDCl}_{3}, 400 \mathrm{MHz}\right) \delta: 8.37-8.26(\mathrm{~m}, 2 \mathrm{H}), 7.88-7.85(\mathrm{~m}, 2 \mathrm{H}), 7.43-7.39(\mathrm{~m}, 2 \mathrm{H})$, $7.03(\mathrm{t}, 2 \mathrm{H}, J=8.8 \mathrm{~Hz}), 6.44(\mathrm{~s}, 1 \mathrm{H}), 3.42(\mathrm{~d}, 1 \mathrm{H}, J=18.8 \mathrm{~Hz}), 3.25(\mathrm{dd}, 1 \mathrm{H}, J=2.4$, $18.8 \mathrm{~Hz}), 2.35(\mathrm{~s}, 2 \mathrm{H}), 1.27-1.22(\mathrm{~s}, 6 \mathrm{H}) ;{ }^{13} \mathrm{C} \mathrm{NMR}\left(\mathrm{CDCl}_{3}, 100 \mathrm{MHz}\right) \delta: 192.1,163.9$, $161.5,156.0,154.4,151.0,134.6,133.6,132.2,129.0,128.9,128.0,127.7,118.2,115.8$, 115.6, 64.3, 50.9, 38.0, 34.6, 28.7, 28.4; MS (ESI) $\mathrm{m} / \mathrm{z} 391(\mathrm{M}+1)$; Anal. calcd for $\mathrm{C}_{23} \mathrm{H}_{19} \mathrm{FN}_{2} \mathrm{O}_{3:}$ C 70.76, H 4.91, N 7.18; found: C 70.82, H 4.88, N 7.26.

3,4-Dihydro-3,3-dimethyl-13-(2-chlorophenyl)-2H-indazolo[2,1-b]phthalazine-1,6, 11(13H)-trione $(\mathbf{4 h})$

${ }^{1} \mathrm{H}$ NMR $\left(\mathrm{CDCl}_{3}, 400 \mathrm{MHz}\right) \delta: 8.39-8.25(\mathrm{~m}, 2 \mathrm{H}), 7.89-7.84(\mathrm{~m}, 2 \mathrm{H}), 7.49(\mathrm{~d}, 1 \mathrm{H}$, $J=6.8 \mathrm{~Hz}), 7.34-7.22(\mathrm{~m}, 3 \mathrm{H}), 6.69(\mathrm{~s}, 1 \mathrm{H}), 3.42(\mathrm{~d}, 1 \mathrm{H}, J=18.8 \mathrm{~Hz}), 3.25(\mathrm{dd}, 1 \mathrm{H}, J=2.0$, $18.8 \mathrm{~Hz}), 2.33(\mathrm{~s}, 2 \mathrm{H}), 1.27-1.22(\mathrm{~m}, 6 \mathrm{H}) ;{ }^{13} \mathrm{C} \mathrm{NMR}\left(\mathrm{CDCl}_{3}, 100 \mathrm{MHz}\right) \delta: 192.1,156.2$, $154.2,151.8,134.5,133.6,133.0,132.5,130.5,129.9,129.0,128.7,128.0,127.7,127.2$, 64.1, 50.8, 38.0, 34.6, 28.8, 28.4; MS (ESI) $\mathrm{m} / \mathrm{z} 407(\mathrm{M}+1)$; Anal. calcd for $\mathrm{C}_{23} \mathrm{H}_{19} \mathrm{ClN}_{2} \mathrm{O}_{3}$ : C 67.90, H 4.71, N 6.89; found: C 70.02, H 4.69, N 6.95.

3,4-Dihydro-3,3-dimethyl-13-(2,4-dichlorophenyl)-2H-indazolo[2,1-b]phthalazine-1,6, 11(13H)-trione (4i)

${ }^{1} \mathrm{H}$ NMR $\left(\mathrm{CDCl}_{3}, 400 \mathrm{MHz}\right) \delta: 8.38-8.24(\mathrm{~m}, 2 \mathrm{H}), 7.90-7.86(\mathrm{~m}, 2 \mathrm{H}), 7.43(\mathrm{~d}, 2 \mathrm{H}$, $J=8.0 \mathrm{~Hz}), 7.35-7.27(\mathrm{~m}, 2 \mathrm{H}), 6.64(\mathrm{~s}, 1 \mathrm{H}), 3.40(\mathrm{~d}, 1 \mathrm{H}, J=18.8 \mathrm{~Hz}), 3.25(\mathrm{dd}, 1 \mathrm{H}, J=2.4$, 
$18.8 \mathrm{~Hz}), 2.38-2.29(\mathrm{~m} 2 \mathrm{H}), 1.23-1.21(\mathrm{~m}, 6 \mathrm{H}) ;{ }^{13} \mathrm{C} \mathrm{NMR}\left(\mathrm{CDCl}_{3}, 100 \mathrm{MHz}\right) \delta: 192.1$, $156.1,154.3,152.1,135.1,134.6,133.7,131.7,130.4,129.0,128.5,128.1,127.7,127.6$, $116.1,64.2,50.8,38.0,34.6,28.8,28.4$; MS (ESI) $\mathrm{m} / \mathrm{z} 441(\mathrm{M}+1)$; Anal. calcd for $\mathrm{C}_{23} \mathrm{H}_{18} \mathrm{Cl}_{2} \mathrm{~N}_{2} \mathrm{O}_{3}$ : C 62.60, H 4.11, N 6.35; found: C 62.76, H 4.02, N 6.48.

3,4-Dihydro-3,3-dimethyl-13-(3,4-dichlorophenyl)-2H-indazolo[2,1-b]phthalazine-1,6, 11(13H)-trione $(\mathbf{4 j})$

${ }^{1} \mathrm{H}$ NMR $\left(\mathrm{CDCl}_{3}, 400 \mathrm{MHz}\right) \delta: 8.39-8.27(\mathrm{~m}, 2 \mathrm{H}), 7.90-7.87(\mathrm{~m}, 2 \mathrm{H}), 7.46-7.42(\mathrm{~m}, 2 \mathrm{H})$, $7.32(\mathrm{dd}, 1 \mathrm{H}, J=2.0,7.6 \mathrm{~Hz}), 6.39(\mathrm{~s}, 1 \mathrm{H}), 3.41(\mathrm{~d}, 1 \mathrm{H}, J=19.2 \mathrm{~Hz}), 3.25(\mathrm{dd}, 1 \mathrm{H}, J=1.6$, $19.2 \mathrm{~Hz}), 2.35$ (s 2H) $1.27-1.22(\mathrm{~s}, 6 \mathrm{H}) ;{ }^{13} \mathrm{C} \mathrm{NMR}\left(\mathrm{CDCl}_{3}, 100 \mathrm{MHz}\right) \delta: 192.0,155.9$, $154.5,151.4,136.6,134.7,133.8,133.0,132.8,130.7,128.9,128.8,128.7,128.1$, 127.7, 126.8, 117.5, 63.8, 50.8, 38.0, 34.7, 28.6, 28.5; MS (ESI) $\mathrm{m} / \mathrm{z} 441(\mathrm{M}+1)$; Anal. calcd for $\mathrm{C}_{23} \mathrm{H}_{18} \mathrm{Cl}_{2} \mathrm{~N}_{2} \mathrm{O}_{3}$ : $62.60, \mathrm{H}$ 4.11, N 6.35; found: C 62.65, H 4.23, N 6.30.

\section{3,4-Dihydro-3,3-dimethyl-13-(3,4,5-trimethoxyl)-2H-indazolo[2,1-b]phthalazine-1,6, $11(13 H)$-trione $(\mathbf{4 k})$}

${ }^{1} \mathrm{H} \mathrm{NMR}\left(\mathrm{CDCl}_{3}, 400 \mathrm{MHz}\right) \delta: 8.38-8.30(\mathrm{~m}, 2 \mathrm{H}), 7.89-7.87(\mathrm{~m}, 2 \mathrm{H}), 6.64(\mathrm{~s}, 2 \mathrm{H}), 6.40$ (s, $1 \mathrm{H}), 3.83-3.81(\mathrm{~m}, 9 \mathrm{H}), 3.46(\mathrm{~d}, 1 \mathrm{H}, J=18.8 \mathrm{~Hz}), 3.24(\mathrm{dd}, 1 \mathrm{H}, J=2.0,18.8 \mathrm{~Hz}), 2.37$ (s $2 \mathrm{H}), 1.26-1.24(\mathrm{~s}, 6 \mathrm{H}) ;{ }^{13} \mathrm{C} \mathrm{NMR}\left(\mathrm{CDCl}_{3}, 100 \mathrm{MHz}\right) \delta$ : 192.2, 156.1, 154.5, 153.3, 150.8, 138.2, 134.6, 133.6, 131.8, 129.0, 128.9, 128.0, 127.7, 1183.3, 104.6, 65.0, 60.7, 56.2, 50.9, 38.1, 34.6, 29.7, 28.9, 28.1; MS (ESI) $m / z, 463(\mathrm{M}+1)$; Anal. calcd for $\mathrm{C}_{26} \mathrm{H}_{26} \mathrm{~N}_{2} \mathrm{O}_{6:}$ C 67.52, H 5.67, N 6.06; found: C 67.62, H 5.74, N 6.01.

\section{Results and Discussion}

In a preliminary study, the effect of amount of the catalyst on the reaction yield of $2 \mathrm{H}$-indazolo $[2,1-b]$ phthalazine-1,6,11(13H)-trione derivatives was investigated with the reaction of dimedone, benzaldehyde and phthalhydrazide as a model reaction in ethanol at reflux temperature. As shown in Table 1, in the absence of catalyst no product was obtained. We found that $10 \mathrm{~mol} \%$ of the catalyst was sufficient to mediate the reaction toward the formation of the corresponding $2 H$-indazolo[2,1- $b$ ]phthalazine-1,6,11(13H)-trione in excellent yield. The lower quantities of the catalyst (i.e. $5 \mathrm{~mol} \%$ ) also gave moderate yield of the product at longer reaction time.

Table 1. The amounts of catalyst optimization for the synthesis of $4 \mathbf{a}^{\mathrm{a}}$

\begin{tabular}{cccc}
\hline Entry & $\mathrm{I}_{2} / \mathrm{mol} \%$ & Time $/ \min$ & Yield $/ \%^{\mathrm{b}}$ \\
\hline 1 & 0 & 120 & 0 \\
2 & 5 & 20 & 62 \\
3 & 10 & 20 & 92 \\
4 & 15 & 20 & 90 \\
5 & 20 & 20 & 92 \\
6 & 25 & 20 & 90 \\
\hline
\end{tabular}

${ }^{a}$ Reaction conditions: dimedone (1 mmol); benzaldehyde $(1.2 \mathrm{mmol}) ;$ phthalhydrazide (1 mmol); EtOH $(10 \mathrm{~mL})$; refiux. ${ }^{b}$ Isolated yield

To find the optimal solvent for this reaction, the synthesis of $\mathbf{4 a}$ was carried out at $80{ }^{\circ} \mathrm{C}$ or reflux temperature using ethanol, $\mathrm{H}_{2} \mathrm{O}, \mathrm{CH}_{2} \mathrm{Cl}_{2}, \mathrm{DMF}$ and $\mathrm{CH}_{3} \mathrm{CN}$ as solvents, respectively. It is shown in Table 2 that the reactions with ethanol as solvent resulted in higher yield than other solvents. So ethanol was chosen as the solvent of this reaction. 
Table 2. Solvent optimization for the synthesis of $\mathbf{4} \mathbf{a}^{\mathrm{a}}$

\begin{tabular}{ccccc}
\hline Entry & Solvent & Temperature $/{ }^{\circ} \mathrm{C}$ & Time $/$ min & Yield $/ \%^{b}$ \\
\hline 1 & Ethanol & reflux & 30 & 92 \\
2 & $\mathrm{H}_{2} \mathrm{O}$ & 80 & 120 & 8 \\
3 & $\mathrm{CH}_{2} \mathrm{Cl}_{2}$, & reflux & 60 & 62 \\
4 & $\mathrm{DMF}$ & 80 & 60 & 52 \\
5 & $\mathrm{CH}_{3} \mathrm{CN}$ & 80 & 60 & 56 \\
\hline
\end{tabular}

${ }^{a}$ Reaction conditions: dimedone $(1 \mathrm{mmol})$; benzaldehyde $(1.2 \mathrm{mmol})$; phthalhydrazide $(1 \mathrm{mmol}) ; \mathrm{I}_{2}$ (1 mmol). ${ }^{b}$ Isolated yield

Based on the optimized reaction conditions, a range of $2 H$-indazolo[2,1- $b]$ phthalazine1,6,11(13H)-trione derivatives (4) was synthesized by the reaction of dimedone $(\mathbf{1}, 1 \mathrm{mmol})$, aromatic aldehydes $(2,1 \mathrm{mmol})$ and phthalhydrazide $(3,1 \mathrm{mmol})$. The reaction proceeded at reflux within $30 \mathrm{~min}$ in excellent yields after the addition of $10 \mathrm{~mol} \% \mathrm{I}_{2}$. Table 3 shows that both electron-deficient and electron-rich aromatic aldehydeswere converted to the corresponding $2 \mathrm{H}$-indazolo[2,1-b]phthalazine- 1,6,11(13H)-trione derivatives in moderate yields. The structures of the products were established from their spectral properties $\left({ }^{1} \mathrm{H}\right.$ NMR, ${ }^{1} \mathrm{C}$ NMR and elemental analysis).

Table 3. Synthesis of $2 H$-indazolo[2,1-b]phthalazine-1,6,11(13H)-trione derivatives ${ }^{a}$

\begin{tabular}{lcccc}
\hline Entry & $\mathrm{R}$ & Time $/$ min & Yield $/ \%^{\mathrm{b}}$ & m.p. $/{ }^{\circ} \mathrm{C}$ (Lit. m.p.) [ref.] \\
\hline $\mathbf{a}$ & $\mathrm{C}_{6} \mathrm{H}_{5}$ & 20 & 93 & $205-207(204-206))^{[10]}$ \\
$\mathbf{b}$ & $4-\mathrm{Cl}-\mathrm{C}_{6} \mathrm{H}_{4}$ & 20 & 91 & $262-264(258-260)^{[10]}$ \\
$\mathbf{c}$ & $4-\mathrm{MeO}-\mathrm{C}_{6} \mathrm{H}_{4}$ & 10 & 96 & $220-221(218-220)^{[9]}$ \\
$\mathbf{d}$ & $4-\mathrm{Me}-\mathrm{C}_{6} \mathrm{H}_{4}$ & 10 & 95 & $228-230(226-231)^{[10]}$ \\
$\mathbf{e}$ & $4-\mathrm{NO}_{2}-\mathrm{C}_{6} \mathrm{H}_{4}$ & 20 & 90 & $220-222(216-218)^{[10]}$ \\
$\mathbf{f}$ & $3-\mathrm{NO}_{2}-\mathrm{C}_{6} \mathrm{H}_{4}$ & 30 & 87 & $270-270(269-271)^{[10]}$ \\
$\mathbf{g}$ & $4-\mathrm{F}_{6} \mathrm{H}_{4}$ & 25 & 89 & $220-220-2(221-223)^{[10]}$ \\
$\mathbf{h}$ & $2-\mathrm{Cl}_{6}-\mathrm{C}_{6} \mathrm{H}_{4}$ & 25 & 90 & $262-264(266-269){ }^{[10]}$ \\
$\mathbf{i}$ & $2,4-\mathrm{Cl}_{2}-\mathrm{C}_{6} \mathrm{H}_{3}$ & 15 & 93 & $222-224(218-220)^{[10]}$ \\
$\mathbf{j}$ & $3,4-\mathrm{Cl}_{2}-\mathrm{C}_{6} \mathrm{H}_{3}$ & 15 & 94 & $262-264$ \\
$\mathbf{k}$ & $3,4,5-\mathrm{MeO}^{2} \mathrm{C}_{6} \mathrm{H}_{2}$ & 30 & 86 & $233-235(232-234){ }^{[10]}$ \\
\hline
\end{tabular}

${ }^{a}$ Reaction conditions: dimedone $(1 \mathrm{mmol})$; aldehyde $(1.2 \mathrm{mmol})$; phthalhydrazide $(1 \mathrm{mmol}) ; \mathrm{I}_{2}$ ( $0.1 \mathrm{mmol})$; ethanol; reflux. ${ }^{b}$ Isolated yield

The plausible mechanism of the reaction is shown in Scheme 2. It is conceivable that molecular iodine is capable of binding with the carbonyl oxygen increasing the reactivities of parent carbonyl as it behaves as a mild lewis acid. First molecular iodine activates carbonyl group of aromatic aldehyde to give iodine-aldehyde complex $\mathbf{I}$ and thus increases the electrophilicity carbonyl carbon of aldehyde. Nuleophilic addition of dimedone to I to give II and followed by loss of $\mathrm{H}_{2} \mathrm{O}$ from II to afford III, which is further activated by iodine. Subsequent Michael-type addition of phthalhydrazide to the olefin afford the corresponding products $\mathbf{4 a - 4 k}$.

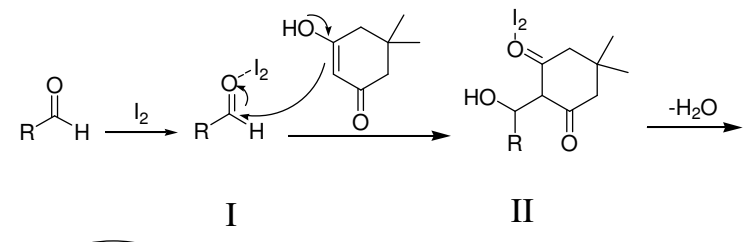




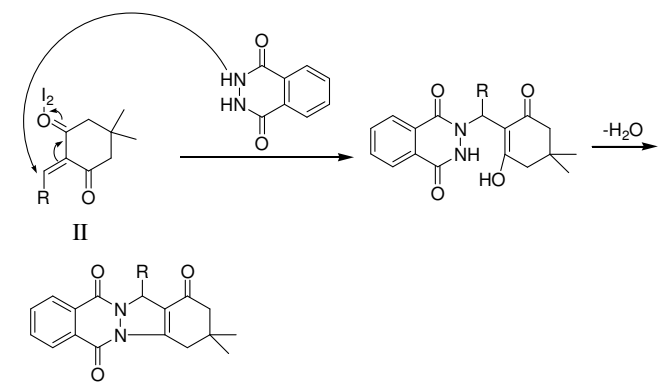

\section{Conclusion}

Scheme 2

An efficient methodoly for the synthesis of $2 H$-indazolo[2,1- $b]$ phthalazine-1,6,11(13H)trione derivatives has been developed by multicomponent condensation of dimedone, aromatic aldehydes and phthalhydrazide in the presence of molecular iodine as a catalyst in ethanol. The simple experimental procedure, utilization of an inexpensive and readily available catalyst and excellent yields are the advantages of the present method.

\section{Acknowledgment}

We are pleased to acknowledge the financial support from Xinxiang Medical University.

\section{Reference}

1. El-Sakka S S, Soliman A H and Imam A M, Afinidad, 2009, 66, 167.

2. Zhang L, Guan L P, Sun X Y, Wei C X, Chai K Y and Quan Z S, Chem Bio Drug Design, 2009, 73, 313.

3. Ryu C-K, Park R-E, Ma M-Y and Nho J-H, Bioorg Med Chem Lett., 2007, 17, 2577-2580.

4. Li J, Zhao Y F, Yuan X Y, Xu J X and Gong P, Molecules, 2006, 11, 574-582.

5. Sinkkonen J, Ovcharenko V, Zelenin K N, Bezhan I P, Chakchir B A, Al-Assar F and Pihlaja K, Eur J Org Chem., 2002, 2046-2053.

6. (a) Ghahremanzadeh R, Shakibaei G I and Bazgir A, Synlett., 2008, 1129; (b) Nabid M R, Rezaei S J T, Ghahremanzadeh R and Bazgir A, Ultrason Sonochem., 2010, 17, 159; (c) Ghahremanzadeh R, Ahadi S, Sayyafi M and Bazgir A, Tetrahedron Lett., 2008, 49, 4479-4482; (d) Liu L-P, Lu J-M and Shi M, Org Lett., 2007, 9, 1303.

7. Sayyafi M, Seyyedhamzeh M, Khavasi H R and Bazgir A, Tetrahedron, 2008, 64, 2375-2378.

8. Nagarapu L, Bantu R and Mereyala H B, J Heterocycl Chem., 2009, 46, 728.

9. Shaterian H R, Ghashang M and Feyzi M, Appl Catal A: Gen., 2008, 345, 128.

10. Khurana J M and Magoo D, Tetrahedron Lett., 2009, 50, 7300.

11. Shaterian H R, Khorami F, Amirzadeh A, Doostmohammadi R and Ghashang M, $J$ Iran Chem Res., 2009, 2, 57.

12. Shaterian H R, Hosseinian A and Ghashang M, Arkivoc, 2009, (ii), 59-67.

13. (a) Yadav J S, Reddy B V S, Reddy M S and Prasad A R, Tetrahedron Lett., 2002, 43, 9703; (b) Bandgar B P and Shaikh KA, Tetrahedron Lett., 2003, 44, 1959-1961; (c) Saeeng R, Sirion U, Sahakitpichan P and Isobe M, Tetrahedron Lett., 2003, 44, 6211-6214; (d) Mori N and Togo H, Synlett., 2004, 880-882; (e) Banik B K, Fernandez M, Alvarez C, Tetrahedron Lett., 2005, 46, 2479-2482.

14. (a) Wu L Q, Niu B X, Li W L and Yan F L, Bull Kor Chem Soc., 2009, 30, 2777; (b) Wu L Q, Yang L M, Yan F L, Yang C G and Fang L Z, Bull Korean Chem Soc., 2010, 31, 1051 . 


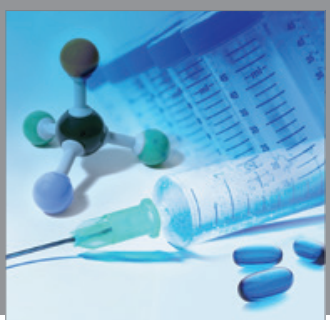

International Journal of

Medicinal Chemistry

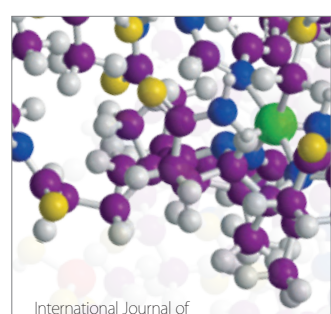

Carbohydrate Chemistry

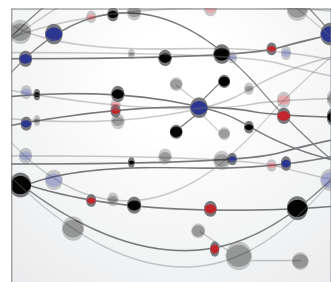

The Scientific World Journal
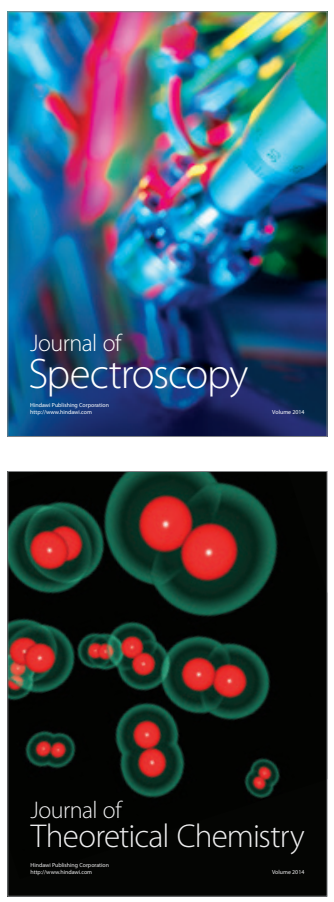
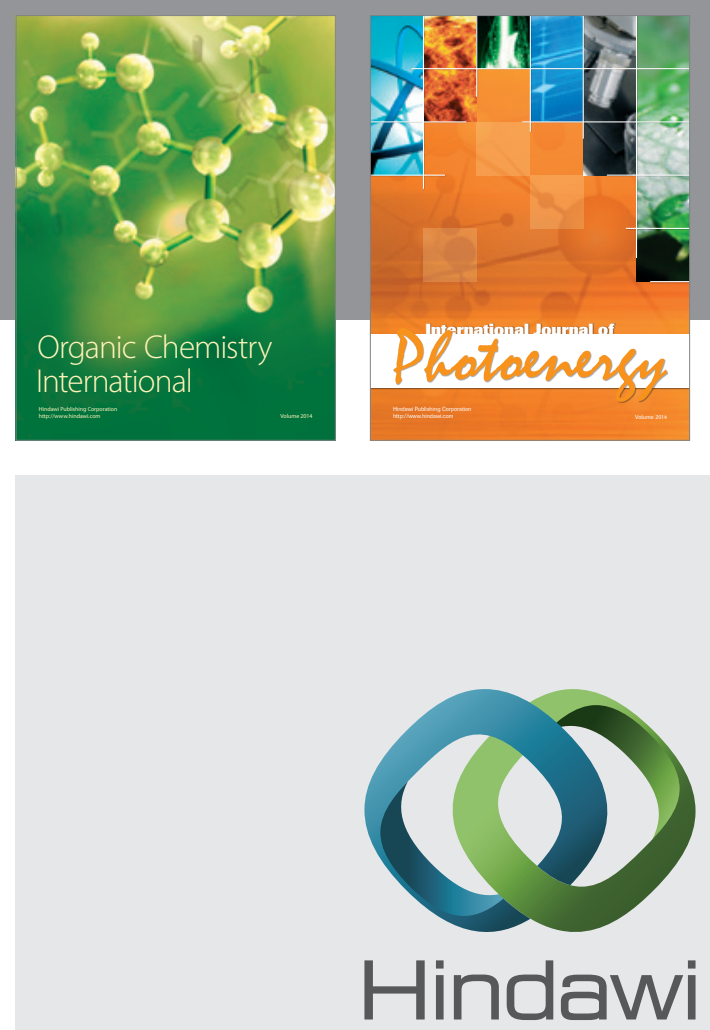

Submit your manuscripts at

http://www.hindawi.com
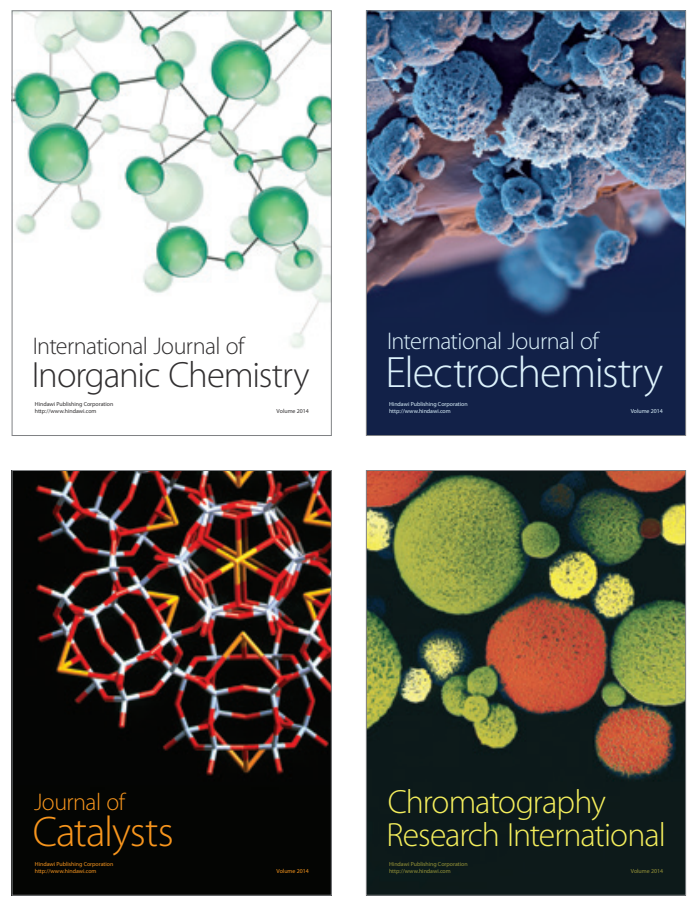
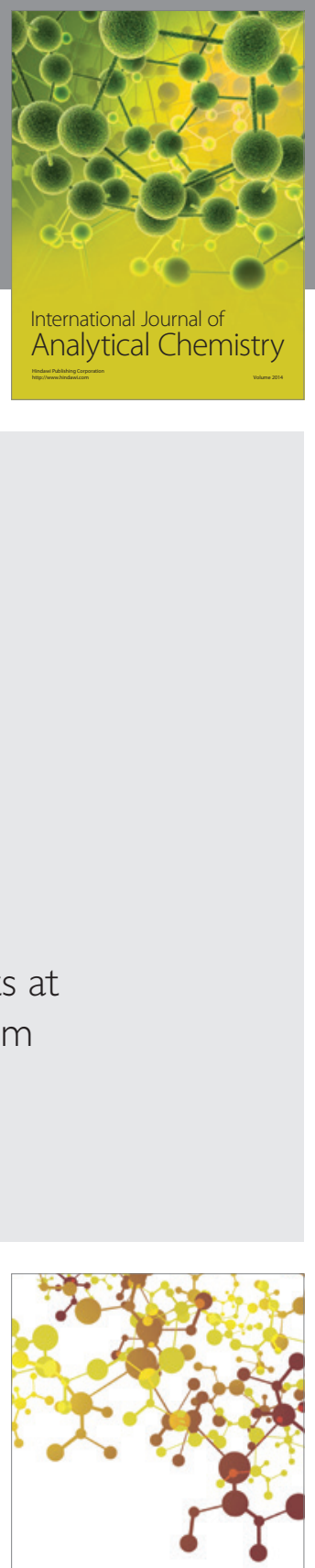

Journal of

Applied Chemistry
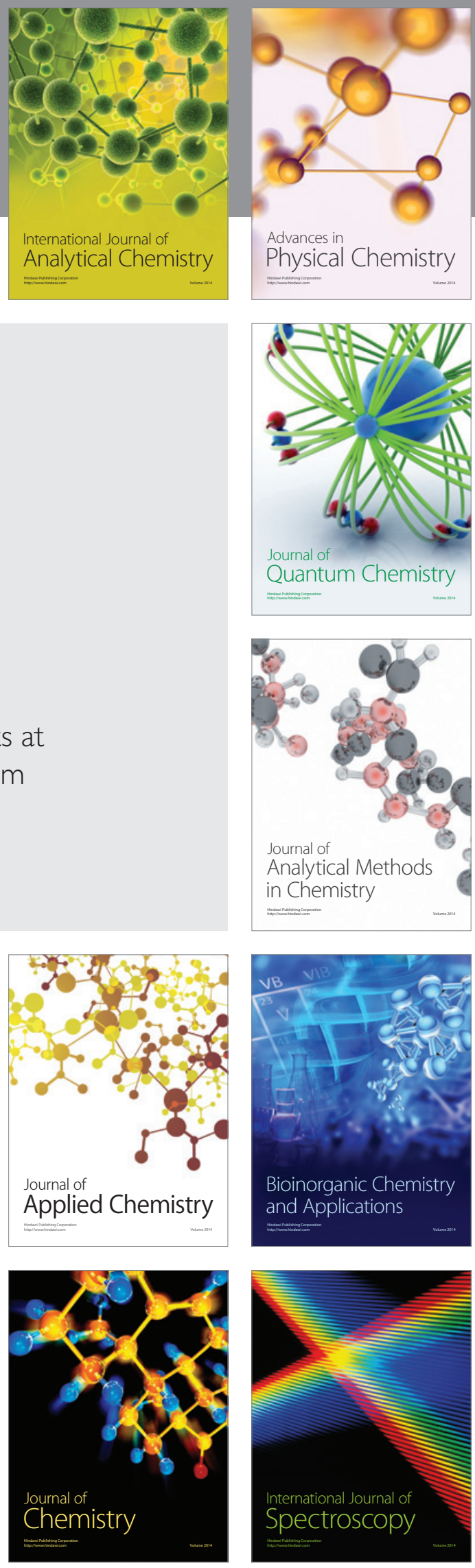